\title{
Structure and Size Determination of the Metal Nanoclusters in the Supported Catalyst: Os3/MgO by Z-Contrast Imaging
} \author{
S. Mehraeen ${ }^{*}, \dagger$
Browning, ${ }^{*}, \dagger$
}

* Department of Chemical Engineering and Materials Science, University of California-Davis, One Shields Avenue, Davis, CA 95616

${ }^{\dagger}$ Department of Molecular and Cellular Biology, University of California-Davis, One Shields

Avenue, Davis, CA 95616

${ }^{\S}$ Materials Science Division, Oak Ridge National Laboratory, Oak Ridge, TN 37830

*Condensed Matter and Materials Division, Lawrence Livermore National Laboratory, Livermore, CA 94550

Supported metal clusters in the form of stabilized nanoparticles [1] have generated wide interest because they offer new and unique structures, reactivities, and catalytic properties that originate from their small sizes and interactions with the support; the metals are typically group- 8 and the supports are typically porous oxides or carbon. The structural information such as cluster uniformity in catalyst determines structure-reactivity relationships and therefore predicts the catalyst's function. In attempts to reach this goal there are variety of synthesis methods proposed to fabricate uniform supported metal clusters [2].

In this work clusters of heavy transition metal (osmium) and a support with light atoms (MgO) was chosen to investigate by scanning transmission electron microscopy (STEM) to determine the uniformity and frame structure of osmium clusters. This was done by measuring the size and size distribution of such clusters from the images. Measuring the cluster size precisely is very important because it allows us to estimate the number and orientation of metal atoms forming the clusters. Highest-resolution microscopy with aberration corrected STEM come closest to determining the cluster structures when the clusters are smallest, containing only a few metal atoms. However, this technique is limited by the high risk of sample damage caused by the high-intensity electron beam, which can lead to dislocation of the metal atoms from their original position in the clusters and therefore deviate original size and frame of the clusters, and in the severe case can fragment them or cause metal aggregation. Low-dose imaging with conventional STEM assures maintaining the cluster frames but this is at the expense of lower signal-to-noise ratio in images which causes the measurements within errors. To overcome this problem recent approach in image analysis methods $[3,4]$ was applied to the images to allow determination of structural details of the metal frames by introducing a shape-independent value as "effective radius" for a cluster. The results demonstrated a high degree of uniformity of the clusters, with effective radii of $0.203 \pm 0.006 \mathrm{~nm}$ matching the theoretical value of $0.202 \mathrm{~nm}$ for a triosmuim triangle cluster where the Os-Os distance is $0.288 \mathrm{~nm}$. Fig. 1a shows the Z-contrast image of the catalyst and Fig. 1b shows the size distribution of the osmium clusters. In a further attempt the sample was imaged by the aberration corrected microscope. The high-resolution STEM images showed the individual Os atoms in the clusters, confirming the triangular structures of their frames (Fig. 2a). The Os-Os distance in the triosmium clusters was directly measured from the $2 \mathrm{D}$ projections and was applied to calculate the Os-Os distance in $3 \mathrm{D}$ as $0.280 \pm 0.014 \mathrm{~nm}$. Fig. $2 \mathrm{~b}$ shows the size histogram of the triosmium clusters. 
This research showed the analysis method applied to conventional STEM images can approve the reliability of the results that can directly be determined by aberration corrected STEM images. Also the aberration corrected STEM results practically showed the mathematical approach towards measuring the cluster size in conventional STEM imaging lays in the valid experimental range. In summary, this research showed how the high- and low-resolution STEM methods can provide the most reliable information about cluster structures and how the methods best complement each other for further applications on unknown cluster structures [5].

[1] J-M. Ha, A. Solovyov, A. Katz, Langmuir 25 (2009) 10548.

[2] J.C. Fierro-Gonzalez, S. Kuba, Y. Hao, B.C. Gates, J. Phys. Chem. B 110 (2006) 13326.

[3] N.L. Okamoto, et.al., Journal of Physical Chemistry C 112 (2008) 1759.

[4] B.W. Reed, et.al., Ultraicroscopy 110 (2009) 48.

[5] This work was supported by the National Science Foundation, GOALI Grant CTS-05-00511.
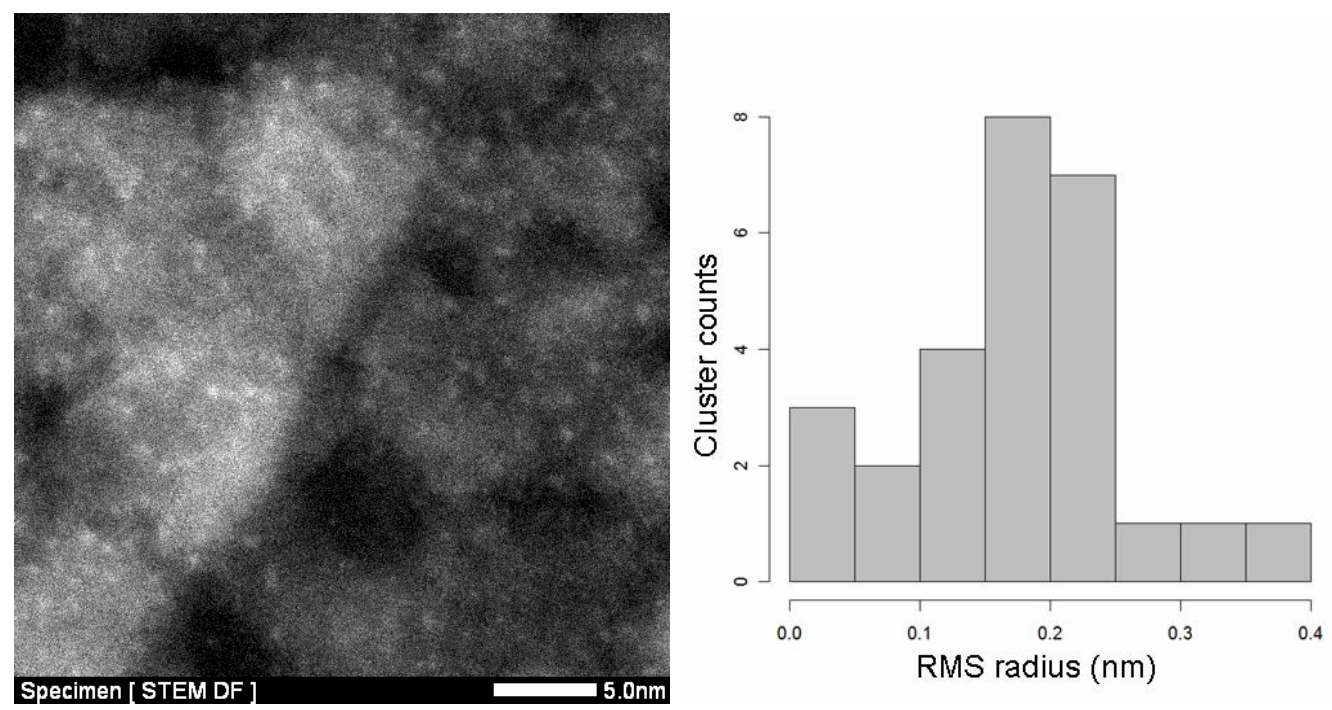

FIG. 1. Supported triosmium clusters, (a) Z-contrast image. (b) Histogram of cluster size.
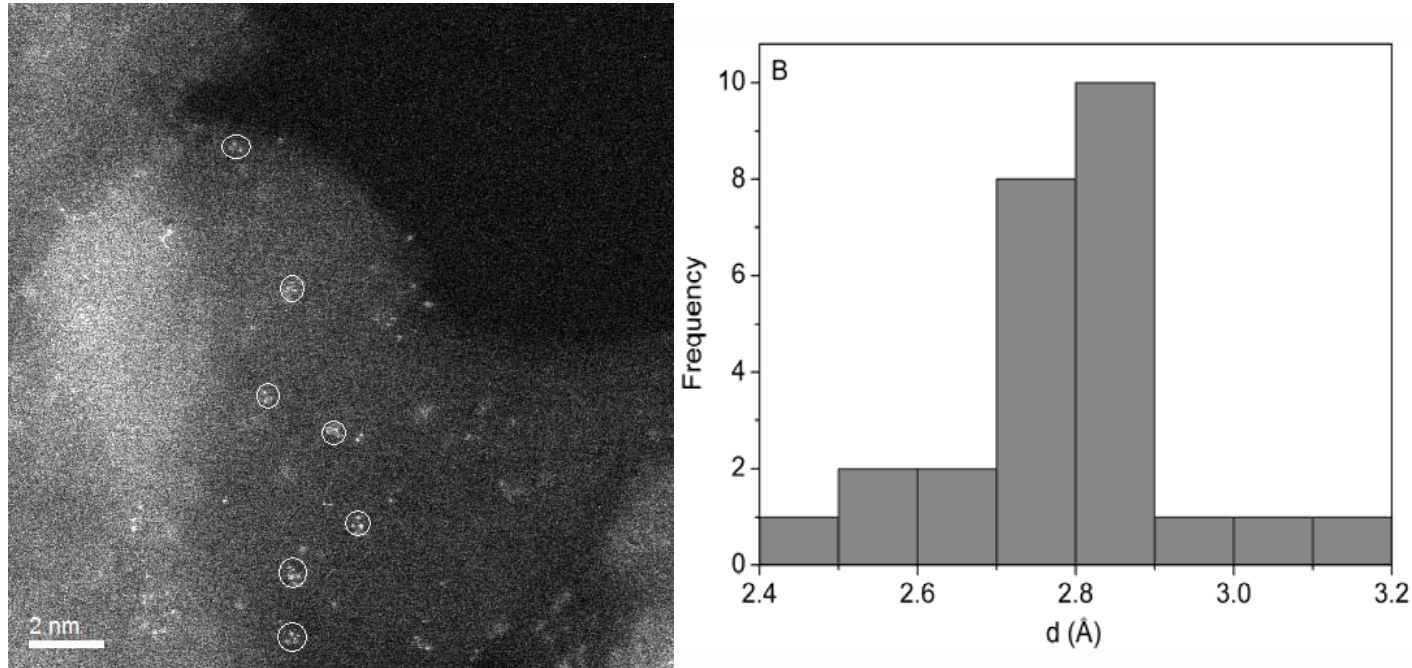

FIG. 2. Supported triosmium clusters, (a) Aberration corrected Z-contrast image. (b) Histogram cluster size. 